\title{
Ligamentoplastie par lambeau périoste péronier pour les laxités externes chroniques de cheville
}

\author{
P. Grumillier
}

Clinique de la Providence, 14, 16, place Ch. de Gaulle, F-55200 Commercy

Les laxités externes chroniques de cheville sont relativement fréquentes et posent des problèmes thérapeutiques difficiles : de nombreuses méthodes ont été proposées mais qui ont pour la plupart le défaut d'utiliser tout ou partie du court péronier latéral. C'est pourquoi la méthode décrite par Roy-Camille et Saillant en 1986 nous a séduit par sa simplicité et par son respect des éléments stabilisateurs actifs de la cheville.

\section{Technique de l'intervention}

L'abord chirurgical est une incision en "J" inversé dont la branche verticale est centrée sur la malléole externe. La découverte et l'exploration des formations capsulaires et ligamentaires se font sans difficulté. Le temps important est donc le prélèvement du lambeau périosté péronier à pédicule inférieur dont la découpe rectangulaire (longueur $8 \mathrm{~cm}$ - largeur $1,5 \mathrm{~cm}$ ) met à nu le plan osseux ce qui est peut être source de troubles douloureux ou trophiques post-opératoires. La base du lambeau sera renforcée au niveau des angles par des points de fil à résorption lente afin d'éviter une fragilisation par les phénomènes de traction. Sur 15 interventions nous avons observé une fois une absence quasi complète de plan périosté ne permettant pas une réalisation efficace du lambeau périosté pédiculé et nous avons dû l'associer à une ligamento- plastie musculaire classique.

La fixation distale du lambeau peut se faire soit par un tunnel trans-osseux astragalien selon la technique décrite par les promoteurs soit plus simplement en le tunnelisant sous l'insertion astragalo-calcanéenne proximale du pédieux. Le geste est ainsi considérablement simplifié et la solidité du montage est suffisante puisque dans notre esprit le lambeau périosté ne représente qu'un apport trophique et non pas un élément de stabilité suffisant à lui même et cette triangulation permet en rapprochant plus ou moins les deux jambages d'obtenir l'effet de tension désiré sur une cheville en position neutre. Les éléments capsulaires et ligamentaires auront bien sûr auparavant été réinsérés ou suturés en paletot selon les techniques classiques. Une immobilisation complémentaire est indispensable et même dans les cas où le périoste est épais de près d'un millimètre la solidité du montage ne permet jamais une mobilisation précoce. Une botte en résine est donc mise en place pour une durée de 4 semaines après laquelle sera entreprise la réducation.

\section{Résultats}

Quinze cas ont été revus avec plus d'un an de recul. Un cas ne peut être inclus dans la série puisqu'il a comporté une ligamentoplastie classique associée. Un cas est un mauvais résultat chez un tra- vailleur lourd victime d'un accident de travail avec des lésions d'arthrose tibio astragalienne débutante et les 13 autres cas ont donné d'excellents résultats sur le plan de la stabilité et de la mobilité. Par contre 3 fois nous avons observé des troubles trophiques avec cicatrice cutanée adhérente et douloureuse au niveau de la zone de prélèvement du lambeau périosté et 2 fois des phénomènes douloureux ont pu faire évoquer un névrome des branches nerveuses du musculo cutané nécessitant des infiltrations à répétition.

\section{Conclusion}

Pour nous l'utilisation du lambeau péronier pédiculé dans les instabilités chroniques externes de cheville est une excellente méthode permettant de respecter tous les éléments stabilisateurs actifs de la cheville. Il faut savoir cependant que cette plastie a surtout un rôle d'apport trophique et peut-être proprioceptif car dans de nombreux cas l'épaisseur du plan périosté est insuffisante pour envisager une solidité permettant de pallier à la disparition des éléments ligamentaires externes.

\section{Bibliographie}

Roy-Camille R, Saillant G, Benazet JP, Feray Ch (1986) Les laxités externes chroniques de la cheville. Cure chirurgicale par une ligamentoplastic au périoste. Rev Chir Orthop 72:121-126 